\title{
Change in bone mineral density as a function of age in women and men and association with the use of antiresorptive agents
}

\author{
Claudie Berger MSc, Lisa Langsetmo PhD, Lawrence Joseph PhD, David A. Hanley MD, \\ K. Shawn Davison PhD, Robert Josse MD, Nancy Kreiger PhD, Alan Tenenhouse MD, \\ David Goltzman MD, and the Canadian Multicentre Osteoporosis Study Research Group
}

$\infty$

See related article page $\mathrm{I} 683$

\section{ABSTRACT}

Background: Measurement of bone mineral density is the most common method of diagnosing and assessing osteoporosis. We sought to estimate the average rate of change in bone mineral density as a function of age among Canadians aged $25-85$, stratified by sex and use of antiresorptive agents.

Methods: We examined a longitudinal cohort of 9423 participants. We measured the bone mineral density in the lumbar spine, total hip and femoral neck at baseline in 1995-1997, and at 3-year (participants aged 40-6o years only) and 5 -year follow-up visits. We used the measurements to compute individual rates of change.

Results: Bone loss in all 3 skeletal sites began among women at age 40-44. Bone loss was particularly rapid in the total hip and was greatest among women aged 50-54 who were transitioning from premenopause to postmenopause, with a change from baseline of $-6.8 \%$ ( $95 \%$ confidence interval $[\mathrm{Cl}]-7.5 \%$ to $-4.9 \%$ ) over 5 years. The rate of decline, particularly in the total hip, increased again among women older than 70 years. Bone loss in all 3 skeletal sites began at an earlier age (25-39) among men than among women. The rate of decline of bone density in the total hip was nearly constant among men 35 and older and then increased among men older than $6_{5}$. Use of antiresorptive agents was associated with attenuated bone loss in both sexes among participants aged 50-79.

Interpretation: The period of accelerated loss of bone mineral density in the hip bones occurring among women and men older than $\sigma_{5}$ may be an important contributor to the increased incidence of hip fracture among patients in that age group. The extent of bone loss that we observed in both sexes indicates that, in the absence of additional risk factors or therapy, repeat testing of bone mineral density to diagnose osteoporosis could be delayed to every 5 years.

Une version française de ce résumé est disponible à l'adresse www.cmaj.ca/cgi/content/full/178/13/1660/DCI

CMAJ 2008;178(13):1660-8
L ow bone mineral density is one of the most important risk factors for fracture..$^{1,2,3-7}$ Treatment with antiresorptive agents has been widely used for several decades, and the results of randomized controlled trials have shown that at least part of their efficacy is associated with their capacity to increase or stabilize bone density. ${ }^{4} \mathrm{Al}$ though clinical guidelines recommend measurement of bone density, among other important risk factors, when assessing a patient's risk for fracture, ${ }^{3,8,9}$ there is no international consensus on the optimal age at which to begin measurement, or on the frequency of measurement. ${ }^{10}$ The Canadian guidelines recommend it for patients aged $6_{5}$ and older, even in the absence of risk factors or treatment, and suggest a frequency of every $2-3$ years. ${ }^{8}$ Furthermore, it has been suggested that the rate of decline rather than a single measurement of bone density may better identify patients with an elevated risk for fracture. ${ }^{11}$ Consequently, determining changes in bone density over time may provide clues on the pathophysiology of fractures and provide more accurate estimates of the optimal timing for repeat measurement.

Previous studies of change in bone mineral density as a function of age have had a number of limitations. Many were cross-sectional; had small samples, limited age ranges or differing inclusion and exclusion criteria; and most excluded men. ${ }^{12-20}$ The third National Health and Nutrition Examination Survey, ${ }^{21}$ a large cross-sectional study based in the United States included women and men aged 20 years and older but excluded only those who were pregnant or who had a fracture in both hips. It reported that, based on a single measurement of bone density in the hip, age-dependent bone loss in the hips begins early (20-40 years) and continues in both sexes

From the CaMos Methods Centre (Berger), McGill University, Montréal, Que.; the CaMos National Coordinating Centre (Langsetmo, Tenenhouse, Kreiger, Goltzman), McGill University, Montréal, Que.; the Department of Epidemiology and Biostatistics (Joseph), McGill University, Montréal, Que.; the Departments of Medicine and Community Health Sciences (Hanley), University of Calgary, Calgary, Alta.; the Department of Rheumatology and Immunology (Davidson), Université Laval, Québec, Que.; the Department of Medicine (Josse), University of Toronto, Toronto, Ont.; the Department of Public Health Sciences (Kreiger), University of Toronto, Toronto, Ont.; the Department of Medicine (Goltzman), McGill University, and the Calcium Research Laboratory, Royal Victoria Hospital, Montréal, Que. 
throughout life. Cross-sectional data from the ongoing Canadian Multicentre Osteoporosis Study suggested that, although this finding may hold true for the femoral neck, which consists of both cortical and trabecular bone, it is not true for the largely trabecular lumbar spine. ${ }^{22}$ Furthermore, the use of cross-sectional data to estimate changes over time has fundamental limitations: the effect of age cannot be separated from the effect of birth cohort and survivorship, and estimates are based on between-group differences rather than changes in an individual participant.

The use of longitudinal data would allow examination of the rate of change of bone mineral density over time with and without antiresorptive therapy. We sought to assess the average rate of change in bone density as a function of age among Canadians aged $25-85$, stratified by sex and use of antiresorptive agents.

\section{Methods}

\section{Participants}

The Canadian Multicentre Osteoporosis Study is an ongoing, prospective cohort study involving 9423 randomly selected community-dwelling women $(n=6539)$ and men $(n=2884)$ aged 25 years and older at baseline and who live within $50 \mathrm{~km}$ of 9 major Canadian cities (St. John's, Newfoundland and Labrador; Halifax, Nova Scotia; Québec, Quebec; Toronto, Hamilton and Kingston, Ontario; Saskatoon, Saskatchewan; Calgary, Alberta; and Vancouver, British Columbia). A detailed description of its purpose, methodology and sampling framework is available elsewhere. ${ }^{23}$ The baseline assessments took place between 1995 and 1997; the 3-year follow-up visits (only for participants aged 40-6o years at baseline) took place between 1998 and 2000; and the 5-year follow-up visits took place between 2000 and 2002. Data collection at baseline and each follow-up visit included an extensive intervieweradministered questionnaire and a clinical assessment. The questionnaire covered sociodemographic information, medical and fracture history, family history, dietary intake, physical activity, tobacco smoking and quality of life. The clinical assessment included recording the participant's height and weight, and measuring his or her bone density by dual energy $\mathrm{x}$-ray absorptiometry of the spine (lumbar vertebrae LI-L4), femoral neck and total hip.

For the present study, we excluded Canadian Multicentre Osteoporosis Study participants who were older than 85 years at baseline ( $2 \%$ of women, I. $6 \%$ of men) because of insufficient sample size. We also excluded those who reported receiving oral or parenteral glucocorticoid therapy for longer than 3 months, either before enrolment or during the 5 years of follow-up, and those who did not have repeated measurements of bone density at I or more of the skeletal sites.

We considered participants to be users of antiresorptive agents if they reported regular use of bisphosphonates, raloxifene, calcitonin or hormone replacement therapy at baseline or during follow-up. We classified women by menopausal status (premenopause v. postmenopause, which we defined as absence of menstrual periods for at least I year or a history of bilateral oophorectomy). We collected these data before the publication of the Women's Health Initiative, ${ }^{24}$ which has had a dramatic impact on the use of hormone replacement therapy. ${ }^{25}$ Of the other antiresorptive agents, cyclical etidronate and alendronate were approved for the treatment of osteoporosis in Canada in 1995, raloxifene in 1998, risedronate and nasal spray salmon calcitonin in 2000.

\section{Bone mineral density measurement}

We measured bone mineral density of the lumbar spine (lumbar vertebrae LI-L4), femoral neck and total hip by dual energy x-ray absorptiometry (QDR machines, Hologic Inc., Waltham, Massachussetts, in 7 centres; or DPX densitometers, GE Lunar, Madison, Wisconsin, in 2 centres). We calibrated the machines daily and performed daily and weekly quality-assurance tests as recommended by the manufacturers. We monitored longitudinal stability using a site-specific spine phantom, and cross-calibrated all densitometers at the start of the study and once each year thereafter using a single European spine phantom. We converted Lunar data into equivalent Hologic values. ${ }^{26}$ One technician reanalyzed all Hologic measurements, and 2 technicians reanalyzed all Lunar measurements. We used the same machine to measure bone density at baseline and at follow-up visits.

\section{Statistical analysis}

We computed slope estimates of bone mineral density measurements over time for individual participants. We used values at baseline and year 5 to compute the change in bone density for $61 \%$ of our sample; values at baseline and years 3 and 5 for about $37 \%$; and values at baseline and year 3 for $2 \%$. For participants who had only 2 measurements, we used the slope between the 2 points to estimate the rate of change. We used a simple linear regression model to estimate the rate of change among those with 3 measurements. For the latter group, we recomputed the change using 2 of the 3 measurements of bone mineral density values (first and third, first and second, second and third). Results for 2-point and 3-point assessments were similar.

We treated age as a categorical variable, with participants grouped in 5-year bands according to age at baseline, and we calculated mean rates of change within these groups. We derived estimates for women and men separately, and we further stratified the estimates by use of antiresorptive agents.

\section{Results}

\section{Participant characteristics}

Of the 9423 participants enrolled in the Canadian Multicentre Osteoporosis Study, 95.5\% (6245/6539) of the women and $96.7 \%(2788 / 2884)$ of the men met our inclusion criteria for age and nonuse of corticosteroids. Of these, we excluded I8I2 women $(29.0 \%)$ and 853 men (30.6\%) because they had no repeat measurements of bone density, which left 6368 participants (4433 women and $1935 \mathrm{men}$ ) for our analysis.

Table I outlines the characteristics at baseline of the participants we included and excluded. We further stratified our sample into users and nonusers of antiresorptive agents. We found that those we excluded generally had more risk factors 


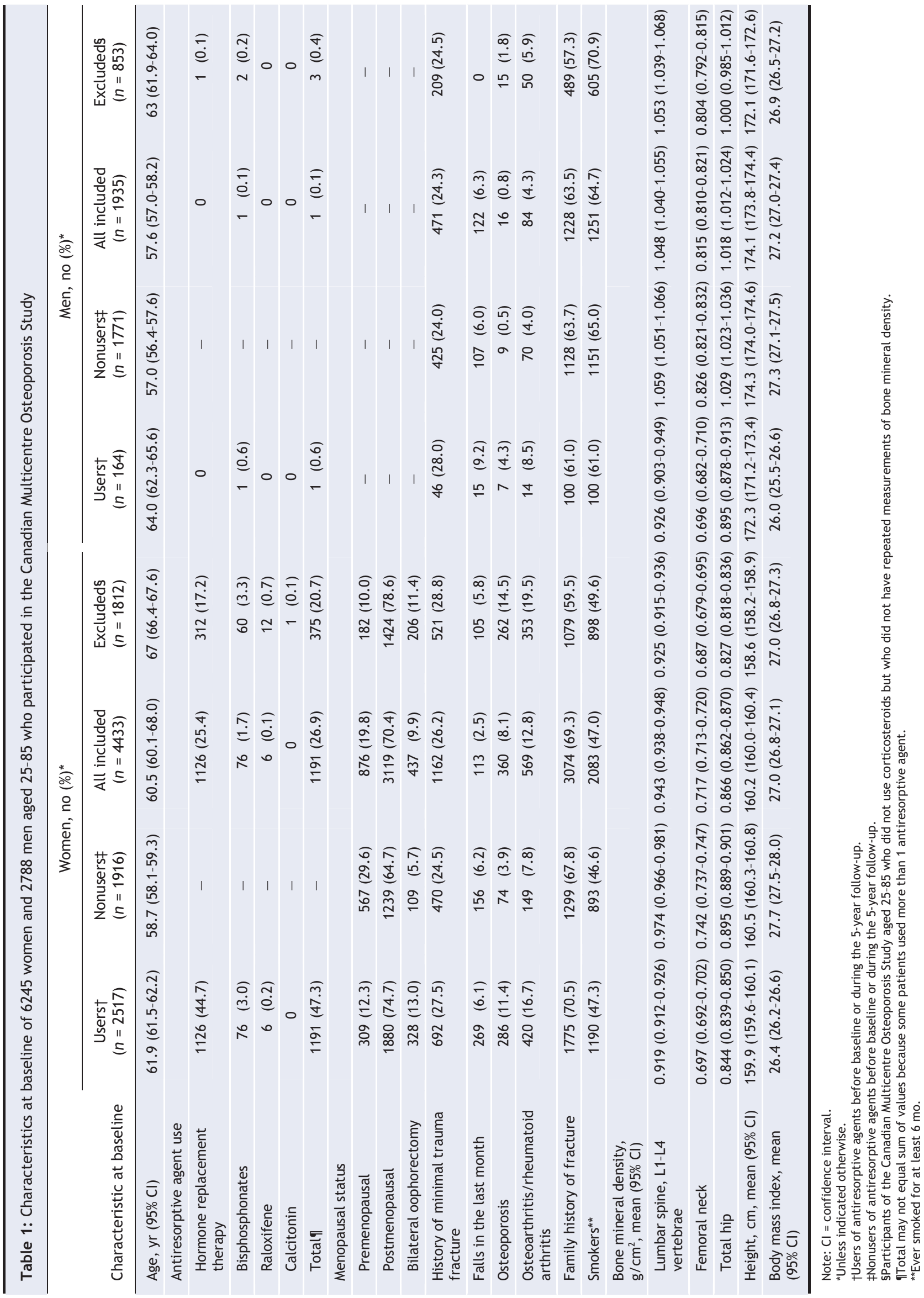


for osteoporotic fractures than did those we included. Excluded people were, on average, $6-7$ years older than those we included, a greater proportion of the excluded women were postmenopausal than premenopausal, and fewer excluded than included individuals were taking hormone replacement therapy. The mean bone density at all skeletal sites tended to be greater among participants than among those excluded. Also, compared with participants, more people in the excluded group had a history of minimal trauma fractures, fewer had a family history of fractures, and more had osteoarthritis and rheumatoid arthritis. In addition, more excluded people than those included reported ever smoking for more than 6 months. The mean body mass index of those included and excluded was the same.

Use of antiresorptive agents at baseline was rare among men (0.1\% among included and $0.4 \%$ among excluded men), compared with $26.9 \%$ among included and $20.7 \%$ among excluded women (Table I). Alendronate was launched in
Canada after the beginning of the baseline data collection period; risedronate became available during year 5 of the study. Consequently, the antiresorptive agent used by the majority of women (both included and excluded) at baseline was hormone replacement therapy. However, after 5 years of follow-up, the percentage of included women who reported regular use of hormone replacement therapy increased from $25.4 \%$ at baseline to $4 \mathrm{I} .6 \%$ at year 5 , and use of bisphosphonates increased from $1.7 \%$ at baseline to $22.0 \%$. Among women who reported using antiresorptive agents at baseline, $93.1 \%$ used hormone replacement therapy alone. However, at year 5 , of the 2517 women who reported using antiresorptive agents at baseline or during follow-up, $56.6 \%$ reported using only hormone replacement therapy, $22.9 \%$ reported using only bisphosphonates and $20.5 \%$ reported using more than one antiresorptive agent (not necessarily at the same time). In year 5,157 of the I 64 men who reported using antiresorptive agents were taking bisphosphonates.
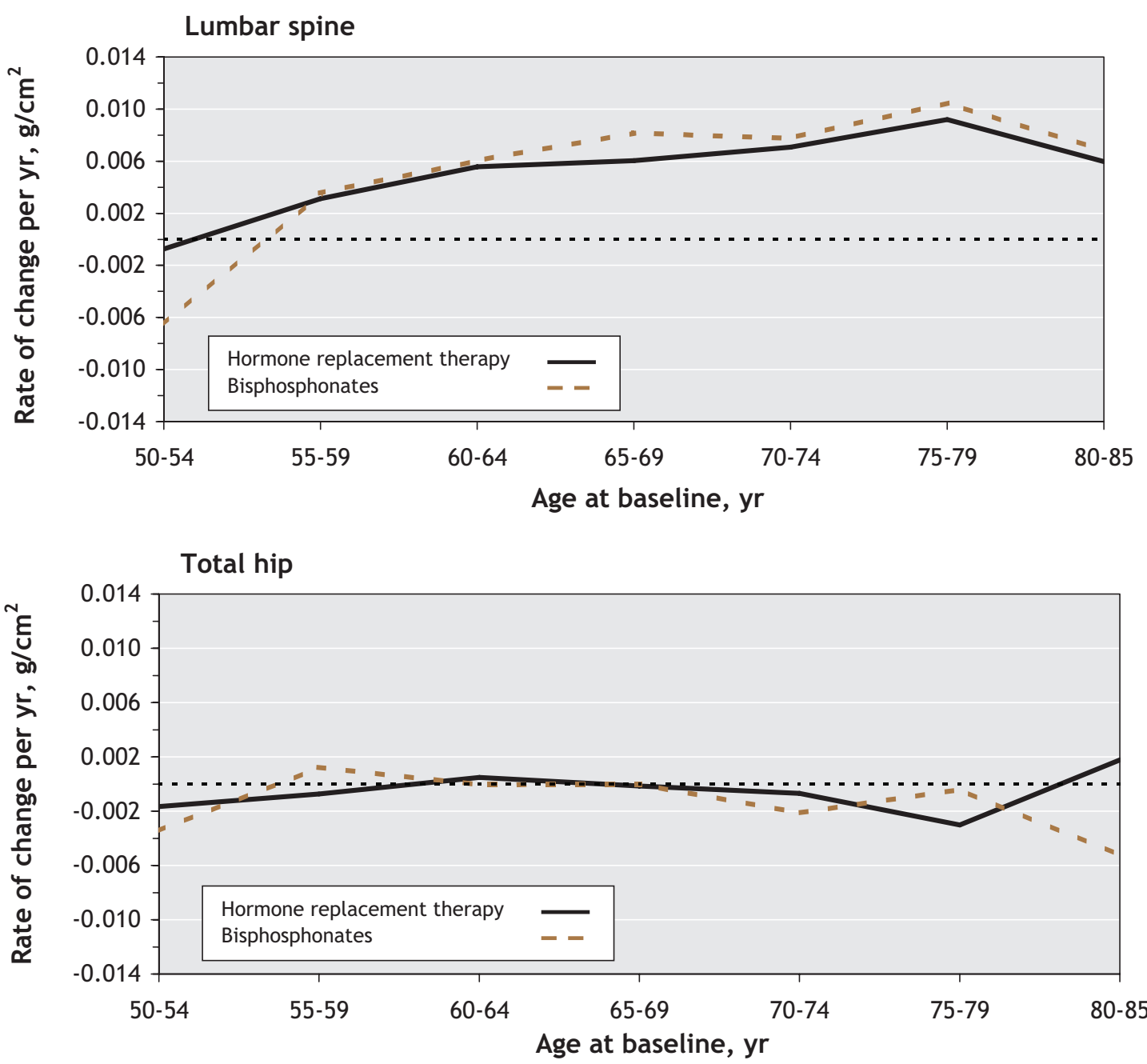

Figure 1: Rate of change in bone mineral density in the lumbar spine (top) and total hip (bottom) as a function of age among women using antiresorptive agents (hormone replacement therapy or bisphosphonates). Bone mineral density is expressed as grams per square centimetre per year. Values above 0 indicate increased bone mineral density, and values below 0 indicate bone loss. 


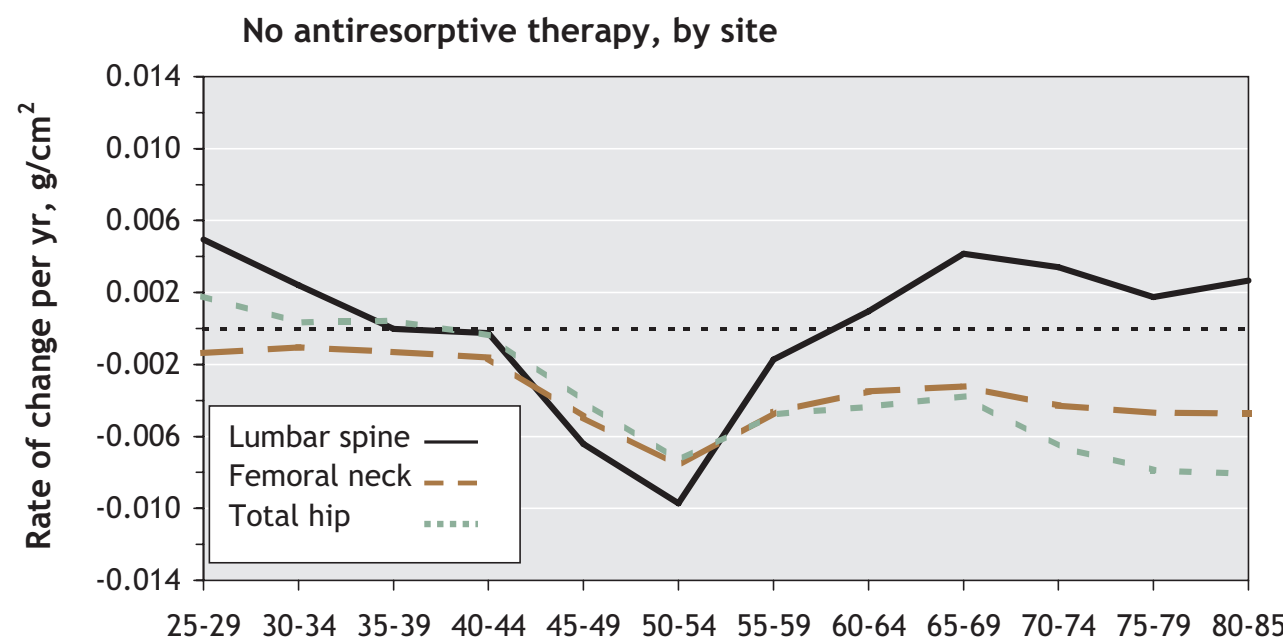

Age at baseline, yr

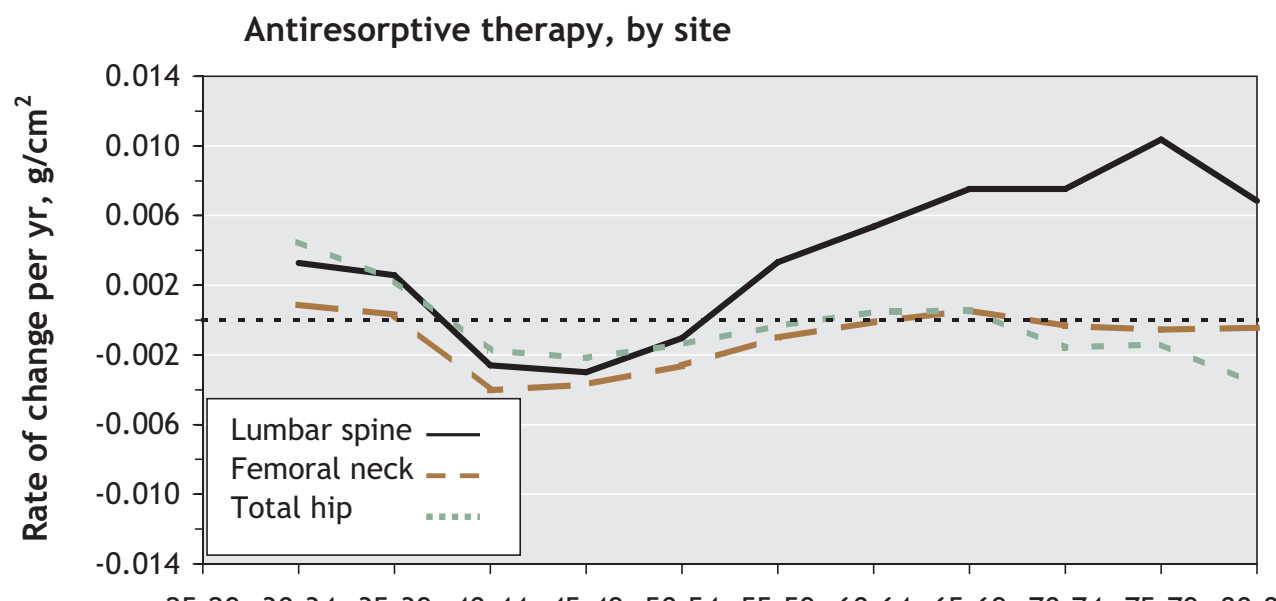

$\begin{array}{llllllllllll}25-29 & 30-34 & 35-39 & 40-44 & 45-49 & 50-54 & 55-59 & 60-64 & 65-69 & 70-74 & 75-79 & 80-85\end{array}$

Age at baseline, yr

No antiresorptive therapy, by menopausal status



Figure 2: Rate of change of bone density as a function of age among women not using (top) or using (middle) antiresorptive agents, and rate of change of bone density in the total hip among women not using antiresorptive agents by menopausal status (bottom). Values above 0 indicate increased bone density, and values below 0 indicate bone loss. 


\section{Change in bone mineral density}

We first compared the change in bone mineral density among women who reported using hormone replacement therapy alone with the change among women who reported using bisphosphonates alone, because these 2 categories account for close to $80 \%$ of those who reported using antiresorptive therapy at baseline or during follow-up. Users of hormone replacement therapy and users of bisphosphonates experienced similar changes in bone density in the lumbar spine and total hip (Figure I). Therefore, we performed further analyses, and results presented are for all antiresorptive agents, irrespective of type.

The estimated mean rates of change per year in bone mineral density in the 3 skeletal sites among women who did not take any antiresorptive agents are shown in Figure 2 (top panel). On average, bone density in the lumbar spine continued to increase (expressed by a positive rate of change) for all participants who were younger than 35 at baseline, with a smaller increase among those aged 30-34. There was no change in mean bone density among women aged 35-44. However, at age 45 bone density began to decline (expressed by a negative rate of change). Women aged 50-54 at baseline reached a maximum mean loss of $-0.0097 \mathrm{~g} / \mathrm{cm}^{2}$ per year ( $95 \%$ confidence interval $[\mathrm{CI}]-0.0 \mathrm{II}$ to -0.0076 ), which corresponded to a change from baseline of $-4.4 \%$ over 5 years ( $95 \%$ CI $-5.5 \%$ to $-3.4 \%$ ). Among women aged 55 and older, the mean rate of decline diminished until, by age $65-69$, there was again a mean increase in bone density in the lumbar spine.

Bone mineral density in the femoral neck and total hip were also relatively stable among women younger than 45 not taking antiresorptive agents (Figure 2, top panel). The mean bone loss at both sites also began at age 45 and reached a maximum rate of decline by age $50-54$. Women aged 55 and older experienced a period of attenuated bone loss; however, by age 70 bone loss accelerated at both sites. The loss of bone mineral density was particularly rapid at the total hip among women aged $70-85$. To better illustrate the effect of peri-
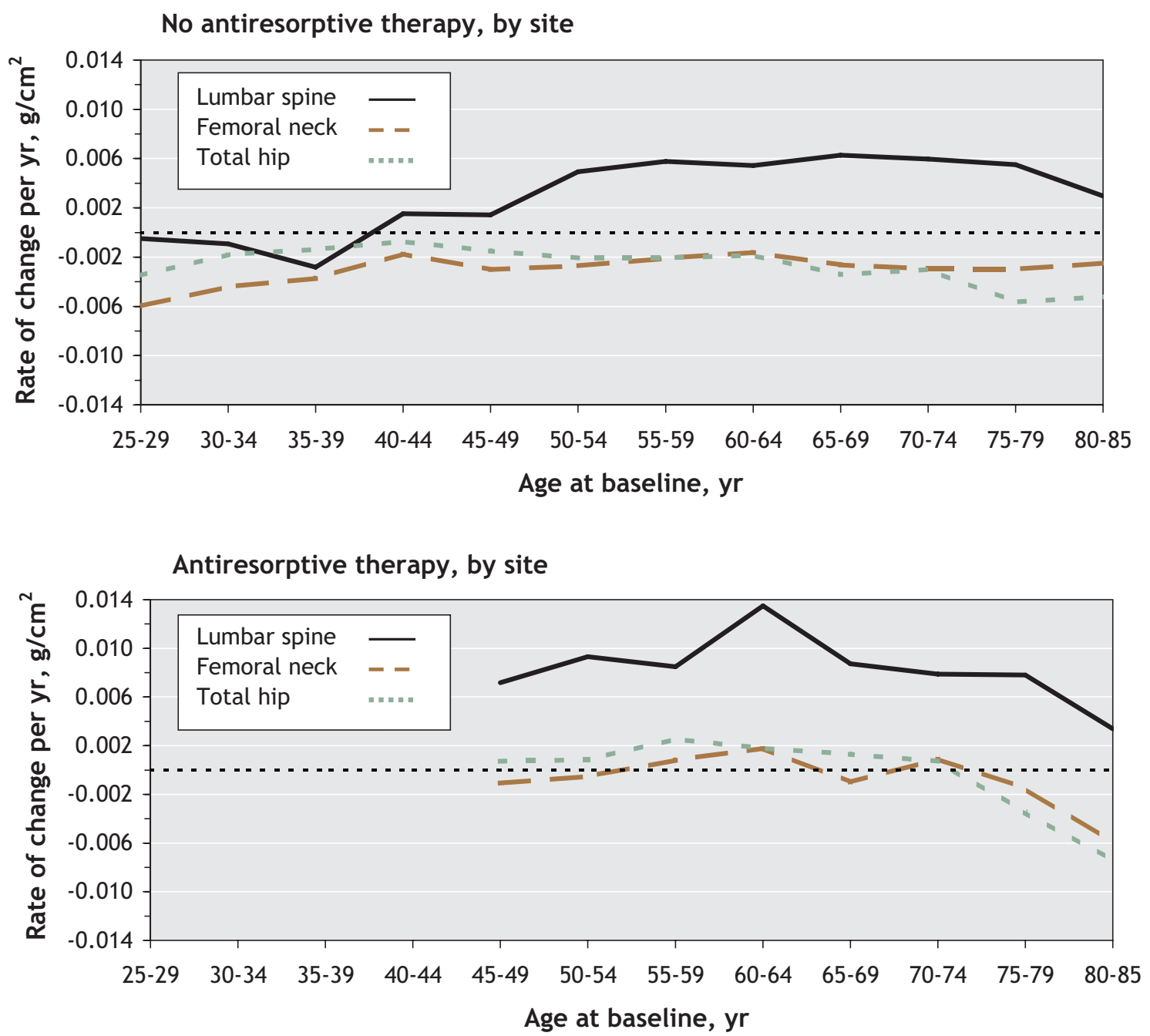

Figure 3: Rate of change of bone density as a function of age among men not using (top) or using (bottom) antiresorptive agents. Values above 0 indicate increased bone density, and values below 0 indicate bone loss. 
menopausal changes on bone loss in women not using antiresorptive agents, we examined the rate of change of bone density in the total hip by menopausal status, i.e., premenopausal (at baseline and at year 5), postmenopausal (at baseline) and in transition (premenopausal at baseline and postmenopausal at year 5) (Figure 2, bottom panel). Women in the transition group experienced the greatest loss: $-0.0122 \mathrm{~g} / \mathrm{cm}^{2}$ per year (95\% CI -0.0150 to -0.0094), which corresponded to a change from baseline of $-6.8 \%$ ( $95 \% \mathrm{CI}$ $-7.5 \%$ to $-4.9 \%$ ) over 5 years.

The middle panel of Figure 2 shows the rates of change in bone mineral density among women using any antiresorptive agent. The mean bone loss in the femoral neck and total hip among women aged 40-55 was markedly attenuated compared with that among women of the same age who did not take antiresorptive agents. Differences between the mean rate of change among users and nonusers of antiresorptive agents older than 50 ranged from $-0.0034 \mathrm{~g} / \mathrm{cm}^{2}(95 \% \mathrm{CI}-0.0045$ to $-0.0023)$ per year in the femoral neck among participants aged 6o-64, which corresponded to a change from baseline of $-2.3 \%$ ( $95 \% \mathrm{CI}-3.1 \%$ to $-\mathrm{I} .5 \%$ ) over 5 years, to $-0.0087 \mathrm{~g} / \mathrm{cm}^{2}$ (95\% CI -0.0III to -0.0062) per year in the lumbar spine among participants aged 50-54, which corresponded to a change from baseline of $-4.0 \%(95 \% \mathrm{CI}-4.8 \%$ to $-3.3 \%$ ) over 5 years. The rate of increase of bone density in the lumbar spine among women older than 60 was augmented by the use of antiresorptive agents: among women aged $70-74$ the rate was $0.0034 \mathrm{~g} / \mathrm{cm}^{2}$ per year among nonusers versus $0.0075 \mathrm{~g} / \mathrm{cm}^{2}$ per year among users, which corresponded to a change from baseline of $\mathrm{r} .8 \%$ versus $4.5 \%$ over 5 years. Among women aged 75-79, the rate was $0.0018 \mathrm{~g} / \mathrm{cm}^{2}$ per year among nonusers versus $0.0104 \mathrm{~g} / \mathrm{cm}^{2}$ per year among users, which corresponded to a change from baseline of $1.0 \%$ versus $6.1 \%$ over 5 years.

Although users of antiresorptive agents had lower bone mineral density at baseline than nonusers had, the comparison of mean changes from baseline among users and nonusers indicated that users had attenuated bone loss. For example, mean changes (over 5 years) from baseline in the lumbar spine among women aged $45-85$ were $0.4 \%$ among nonusers and $2.9 \%$ among users. Mean changes (over 5 years) from baseline in the total hip among women 50-79 were $-3.2 \%$ among nonusers and $-0.2 \%$ among users.

The mean rate of change in bone mineral density as a function of age among men who were not using antiresorptive agents is shown in Figure 3, top panel. In contrast to women, there was a small but significant rate of change in the lumbar spine of $-0.0028 \mathrm{~g} / \mathrm{cm}^{2}$ per year $(95 \% \mathrm{CI}-0.0045$ to -0.0012) among men aged 35-39, which corresponded to a change from baseline of $-\mathrm{I} .3 \%$ ( $95 \% \mathrm{CI}-2 . \mathrm{I} \%$ to $-0.5 \%$ ) over 5 years. Bone density then remained stable until age 50 , after which bone loss resumed. Among men aged 55-59, the mean rate of increase was $0.0058 \mathrm{~g} / \mathrm{cm}^{2}$ per year $(95 \% \mathrm{CI}$ 0.0036 to 0.0080 ), which corresponded to a change from baseline of $2.6 \%$ ( $95 \%$ CI r. $6 \%$ to $3.6 \%$ ) over 5 years; the mean rate of change was similar among men up to age 80 . Bone density in the femoral neck and total hip also decreased at an earlier age (25-39) among men than among women, with a maximum rate of change in the femoral neck of $-0.0060 \mathrm{~g} / \mathrm{cm}^{2}$ per year (95\% CI -0.0088 to -0.0032 ) from age 25 to 29 , which corresponded to a change from baseline of $-3.2 \%$ ( $95 \%$ CI $-4.7 \%$ to $-\mathrm{I} .7 \%$ ) over 5 years. The bone density in the femoral neck and total hip then decreased at a nearly constant rate among men aged 35 or older. The rate of bone loss then appeared to increase after age 65 .

The use of antiresorptive agents among men, as among women, was associated with the rate of bone loss at all skeletal sites studied (Figure 3, bottom panel); however, with few men using antiresorptive agents, the accelerated loss between ages 75 and 85 among the users, and the differences between users and nonusers, were inconclusive.

\section{Interpretation}

Our data document patterns in the rates of accrual and loss of bone mineral density in the lumbar spine and total hip that define differences in skeletal maturation and turnover between women and men. Accelerated loss among women during the perimenopausal period ${ }^{16,18,27,28}$ begins between ages 40 and 44 , peaks between ages 50 and 54 , and then stabilizes. This accelerated loss is a major determinant of the differences in the patterns of bone loss between women and men. A second period of accelerated bone loss begins at age 70 among women, notably in the total hip, which is substantial given that from age 75 years and onward the rate of loss is about the same as at age 50-54. This second period of rapid bone loss among women, and to a possible lesser extent among men (as previously noted by other techniques ${ }^{29}$ ), may be relevant to the increased incidence of hip fractures among elderly patients.

Between ages 25 and 40, women are still gaining or stabilizing bone mass in all skeletal sites, whereas the bone density among men, particularly in the hips, is declining. Nevertheless, in view of the greater incidence of osteoporotic fractures among women, the more profound bone loss around the time of menopause may have greater influence on bone fragility than this early loss among men.

We also observed differences in the temporal pattern of bone loss in the lumbar spine versus the hip sites. This pattern may reflect differences in the composition of bone in the lumbar spine, which has a larger component of trabecular rather than cortical bone than the hips; differences in mechanical stresses in the lumbar spine and the hips; or differences in the development of osteophytosis and other degenerative changes, ${ }^{29}$ which more markedly affect the measurement of vertebral bone density, obscuring the loss of trabecular bone. On average, bone density in the lumbar spine generally either remained stable or increased after age 60 among women or after age 40 among men, whereas bone density in the hips declined. A notable exception to this dichotomous loss was the decline in the lumbar spine among perimenopausal women, which was concordant with the pattern in the hips. Our results for men and women up to ages 50-60 are generally consistent with the findings of others. ${ }^{15,17,19,30,31}$

Our data have important practical clinical implications for the frequency of measuring bone mineral density. Even 
women aged 50-54, among whom we observed the greatest bone loss, experienced a rate of loss of only I. $3 \%$ per year. Although this rate is lower than estimates derived from crosssectional studies ${ }^{32}$ it is consistent with the rates of loss reported among women in other longitudinal studies. ${ }^{15,33}$ However, such values are within the margin of error of most densitometry machines. Although current guidelines recommend that measurements of bone density be repeated once every 2-3 years, ${ }^{8}$ our data suggest that, at this rate of testing, the average person would exhibit changes well below the margin of error, especially since only $25 \%$ of women experienced a loss of bone density that exceeded $5 \%$ over 5 years. Consequently, our data imply that, for more efficient use of health care resources, repeat measurements of bone density could safely be delayed for intervals of up to 5 years unless a therapeutic intervention is being monitored or there are additional clinical risk factors for bone loss, such as corticosteroid use.

We found that use of antiresorptive agents was associated with a reduction in the rate of bone loss at all skeletal sites among participants aged 50 and older. There were few women younger than 45 who used antiresorptive agents, which may explain our inconclusive results for this age group. A gain in bone density in the lumbar spine among women older than 60 was also associated with the use of antiresorptive agents. Inasmuch as such treatment has not been reported to increase degenerative changes and has been reported to reduce osteoarthritis in animal models, ${ }^{34}$ these results may suggest that at least part of the increased bone density that we observed among older women who used antiresorptive agents is not artifactual or solely the result of spinal degenerative changes.

Although our study did show that bone loss attenuated with the use of antiresorptive agents, it is well known that bone loss is only one of several risk factors for fractures. Consequently, the decision to prescribe antiresorptive agents must be based on an assessment of overall risk for fracture, not only on measurements of bone density.

Our study has some limitations. We were unable to determine the dose or duration of treatment with antiresorptive agents, or the degree of adherence to therapy. Furthermore, users and nonusers may have had different underlying patterns of bone loss. Also, in contrast to randomized controlled trials, we included people who used antiresorptive agents with $(33.4 \%$ of female users at year 5$)$ or without a diagnosis of osteoporosis. Although use of antiresorptive agents was clearly associated with a reduced rate of bone loss among women, some of these factors may have led to an underestimate of the reduction that we observed compared with that expected in randomized controlled trials. ${ }^{4}$

In addition, we excluded about $30 \%$ of people who met our inclusion criteria for age and corticosteroid nonuse because they did not have repeat measurements of bone mineral density. On average, our sample was younger and included fewer postmenopausal women and fewer women who were taking hormone replacement therapy or other antiresorptive agents than those excluded. There were clear differences in measured bone density at baseline between those included and excluded, and a greater number of participants in our sample had a family history of fracture compared with those excluded. Therefore, the study population is likely to represent a healthier subpopulation of Canadian women and men, which suggests that the mean bone loss that we observed is an underestimate of the true loss in the population, particularly among the older population.

In summary, our study provides a comprehensive estimate of how bone mineral density changes over time, and it has important physiologic and clinical implications. In addition to marked perimenopausal bone loss, we identified a second stage of bone loss in the hip among elderly women and men. This second decline may predispose the elderly population to increased risk for hip fractures. Furthermore, the extent of the bone loss that we observed suggests that repeat measurements of bone density could be delayed to intervals of up to 5 years in the absence of other risk factors. Finally, the use of antiresorptive agents is associated with attenuation of bone loss among individuals aged 50-79, although introduction of such agents should not be based solely on measurements of bone density.

\section{This article has been peer reviewed.}

Competing interests: Shawn Davison has been paid for research with and has received speaker fees from Proctor \& Gamble, Sanofi-Aventis and Merck Frosst and is a paid consultant on the Servier Bones Strength Board. Robert Josse has served on advisory boards and received honoraria and research grants from Eli Lilly, Proctor \& Gamble, Sanofi-Aventis, Merck Frosst, Novartis, Servier, GlaxoSmithKline and Amgen. David Goltzman has received honoraria from and served on the advisory boards of Amgen, Eli Lilly, Proctor \& Gamble, Merck Frosst, Novartis and Servier. None declared for Claudie Berger, Lisa Langsetmo, Lawrence Joseph, David Hanley, Nancy Kreiger and Alan Tenenhouse.

Contributors: Claudie Berger, Lisa Langsetmo, Lawrence Joseph, Alan Tenenhouse and David Goltzman conceived and designed the study; David Hanley, Shawn Davison, Robert Josse and Nancy Kreiger acquired the data; Claudie Berger and Lawrence Joseph analyzed and interpreted the data; all of the authors drafted or revised the manuscript and approved the final version to be published.

Acknowledgements: We thank all the participants in the Canadian Multicentre Osteoporosis Study.

The Canadian Multicentre Osteoporosis Study was funded by the Canadian Institutes of Health Research (CIHR), Merck Frosst Canada Ltd., Eli Lilly Canada Inc., Novartis Pharmaceuticals Inc., The Alliance for Better Bone Health: Sanofi-Aventis, Procter \& Gamble Pharmaceuticals Canada Inc., The Dairy Farmers of Canada and The Arthritis Society.

\section{REFERENCES}

I. Cummings SR, Black DM, Nevitt MC, et al. Bone density at various sites for prediction of hip fractures. The Study of Osteoporotic Fractures Research Group. Lancet I993;34I:72-5.

2. Schott AM, Cormier C, Hans D, et al. How hip and whole-body bone mineral density predict hip fracture in elderly women: the EPIDOS Prospective Study. Osteoporos Int I998;8:247-54.

3. Kanis JA, Oden A, Johnell $O$, et al. The use of clinical risk factors enhances the performance of BMD in the prediction of hip and osteoporotic fractures in men and women. Osteoporos Int 2007;18:1033-46.

4. Cummings SR. How drugs decrease fracture risk: lessons from trials. J Musculoskelet Neuronal Interact 2002;2:198-200.

5. Melton LJ III, Atkinson EJ, O'Connor MK, et al. Bone density and fracture risk in men. J Bone Miner Res I998;13:1915-23.

6. Miller PD, Siris ES, Barrett-Connor E, et al. Prediction of fracture risk in postmenopausal white women with peripheral bone densitometry: evidence from the National Osteoporosis Risk Assessment. J Bone Miner Res 2002;17:2222-30.

7. van der Klift M, de Laet CE, McCloskey EV, et al. Risk factors for incident vertebral fractures in men and women: the Rotterdam Study. J Bone Miner Res 2004; I9:1172-80. 
8. Brown JP, Josse RG. 2002 clinical practice guidelines for the diagnosis and management of osteoporosis in Canada. CMAJ 2002;I67:SI-34.

9. Osteoporosis prevention, diagnosis, and therapy. NIH Consens Statement 2000;I7:I-45.

Io. Abrahamsen B, Nissen N, Hermann AP, et al. When should densitometry be repeated in healthy peri- and postmenopausal women: the Danish osteoporosis prevention study. J Bone Miner Res 2002;17:206I-7.

II. Nguyen TV, Center JR, Eisman JA. Femoral neck bone loss predicts fracture risk independent of baseline BMD. J Bone Miner Res 2005;20:1195-20I.

I2. Arlot ME, Sornay-Rendu E, Garnero P, et al. Apparent pre- and postmenopausal bone loss evaluated by DXA at different skeletal sites in women: the OFELY cohort. J Bone Miner Res I997;12:683-90.

I3. Szulc P, Marchand F, Duboeuf F, et al. Cross-sectional assessment of age-related bone loss in men: the MINOS study. Bone 2000;26:123-9.

I4. Wu XP, Liao EY, Huang G, et al. A comparison study of the reference curves of bone mineral density at different skeletal sites in native Chinese, Japanese, and American caucasian women. Calcif Tissue Int 2003;73:122-32.

I5. Warming L, Hassager C, Christiansen C. Changes in bone mineral density with age in men and women: a longitudinal study. Osteoporos Int 2002;13:105-I2.

I6. Bainbridge KE, Sowers MF, Crutchfield M, et al. Natural history of bone loss over 6 years among premenopausal and early postmenopausal women. Am J Epidemiol 2002;156:410-7.

I7. Tang GW, Yip PS, Li BY. The profile of bone mineral density in Chinese women: its changes and significance in a longitudinal study. Osteoporos Int 2001;12:647-53

I8. Guthrie JR, Ebeling PR, Hopper JL, et al. A prospective study of bone loss in menopausal Australian-born women. Osteoporos Int 1998;8:282-90.

19. Hui SL, Zhou L, Evans R, et al. Rates of growth and loss of bone mineral in the spine and femoral neck in white females. Osteoporos Int I999;9:200-5.

20. Hou YL, Wu XP, Luo XH, et al. Differences in age-related bone mass of proxima femur between Chinese women and different ethnic women in the United States. $J$ Bone Miner Metab 2007;25:243-52.

2I. Looker AC, Wahner HW, Dunn WL, et al. Updated data on proximal femur bone mineral levels of US adults. Osteoporos Int I998;8:468-89.

22. Tenenhouse A, Joseph L, Kreiger N, et al. Estimation of the prevalence of low bone density in Canadian women and men using a population-specific DXA reference standard: the Canadian Multicentre Osteoporosis Study (CaMos). Osteoporos Int 2000;II:897-904.

23. Kreiger N, Tenenhouse A, Joseph L, et al. Research notes: the Canadian Multicentre Osteoporosis Study (CaMos): background, rationale, methods. Can J Aging I999;18:376-87.

24. Rossouw JE, Anderson GL, Prentice RL, et al. Risks and benefits of estrogen plus progestin in healthy postmenopausal women: principal results from the Women's Health Initiative randomized controlled trial. JAMA 2002;288:32I-33.

25. Majumdar SR, Almasi EA, Stafford RS. Promotion and prescribing of hormone therapy after report of harm by the Women's Health Initiative. JAMA 2004; 292:1983-8

26. Genant HK, Grampp S, Gluer CC, et al. Universal standardization for dual x-ray absorptiometry: patient and phantom cross-calibration results. J Bone Miner Res I994; 9 :1503-I4.

27. Prior JC, Vigna YM, Barr SI, et al. Ovulatory premenopausal women lose cancellous spinal bone: a 5-year prospective study. Bone I996;18:26I-7.

28. Seifert-Klauss V, Link T, Heumann C, et al. Influence of pattern of menopausa transition on the amount of trabecular bone loss. Results from a 6-year prospective longitudinal study. Maturitas 2006;55:317-24.

29. Liu G, Peacock M, Eilam O, et al. Effect of osteoarthritis in the lumbar spine and hip on bone mineral density and diagnosis of osteoporosis in elderly men and women. Osteoporos Int 1997;7:564-9.

30. Lofman O, Larsson L, Ross I, et al. Bone mineral density in normal Swedish women. Bone I997;20:167-74.
3I. Mazess RB, Barden H. Bone density of the spine and femur in adult white females. Calcif Tissue Int I999;65:9I-9.

32. Melton LJ III, Khosla S, Atkinson EJ, et al. Cross-sectional versus longitudinal evaluation of bone loss in men and women. Osteoporos Int 2000; II:592-9.

33. Cauley JA, Lui LY, Stone KL, et al. Longitudinal study of changes in hip bone mineral density in Caucasian and African-American women. J Am Geriatr Soc 2005;53:183-9.

34. Abramson SB, Honig S. Antiresorptive agents and osteoarthritis: More than a bone to pick? Arthritis Rheum 2007;56:2469-73.

Correspondence to: Dr. David Goltzman, Canadian Multicentre Osteoporosis Study, Royal Victoria Hospital, 687 Pine Ave. W, Rm. EI-59, Montréal QC H3A IAI; david.goltzman@mcgill.ca

Canadian Multicentre Osteoporosis Study Research Group: David Goltzman (co-principal investigator), McGill University, Montréal, Que.; Nancy Kreiger (co-principal investigator), University of Toronto, Toronto, Ont.; and Alan Tenenhouse (principal investigator emeritus), Toronto, Ont. Coordinating Centre: Suzette Poliquin (national coordinator), Suzanne Godmaire (research assistant) and Claudie Berger (study statistician), McGill University, Montréal, Que. Study centres: St. John's - Carol Joyce (director), Christopher Kovacs (co-director) and Emma Sheppard (coordinator), Memorial University, St. John's NL. Halifax Susan Kirkland and Stephanie Kaiser (co-directors), and Barbara Stanfield (coordinator), Dalhousie University, Halifax, NS. Québec — Jacques P. Brown (director), Louis Bessette (codirector) and Marc Gendreau (coordinator), Université Laval, Québec, Que. Kingston - Tassos Anastassiades (director), Tanveer Towheed (co-director) and Barbara Matthews (coordinator), Queen's University, Kingston, Ont. Toronto - Bob Josse (director), Sophie Jamal (co-director), Tim Murray (past director) and Barbara Gardner-Bray (coordinator), University of Toronto, Toronto, Ont. Hamilton - Jonathan D. Adachi (director), Alexandra Papaioannou (co-director) and Laura Pickard (coordinator), McMaster University, Hamilton, Ont. Saskatoon Wojciech P. Olszynski (director), K. Shawn Davison (co-director) and Jola Thingvold (coordinator), University of Saskatchewan, Saskatoon, Sask. Calgary - David A. Hanley (director) and Jane Allan (coordinator), University of Calgary, Calgary, Alta. Vancouver - Jerilynn C. Prior (director) and Yvette Vigna (coordinator), University of British Columbia, Vancouver, BC 